\title{
BMJ Global Health The role of HIV viral load in mathematical models of HIV transmission and treatment: a review
}

\author{
Tracy Glass, Landon Myer, Maia Lesosky
}

To cite: Glass T, Myer L, Lesosky M. The role of HIV viral load in mathematical models of HIV transmission and treatment: a review. BMJ Global Health 2020;5:e001800. doi:10.1136/ bmjgh-2019-001800

\section{Handling editor Lei Si}

- Additional material is published online only. To view please visit the journal online (http://dx.doi.org/10.1136/ bmjgh-2019-001800).

Received 28 June 2019 Revised 23 October 2019 Accepted 17 November 2019

Check for updates

(c) Author(s) (or their employer(s)) 2020. Re-use permitted under CC BY-NC. No commercial re-use. See rights and permissions. Published by BMJ.

Division of Epidemiology \& Biostatistics, School of Public Health and Family Medicine, University of Cape Town, Cape Town, South Africa

Correspondence to

Tracy Glass;

Tracy.Glass@uct.ac.za

\section{ABSTRACT}

Introduction HIV viral load (VL) is accepted as a key biomarker in HIV transmission and pathogenesis. This paper presents a review of the role of $V L$ testing in mathematical models for HIV prevention and treatment. Methods A search for simulation models of HIV was conducted in PubMed, yielding a total of 1210 studies. Publications before the year 2000, studies involving animals and analyses that did not use mathematical simulations were excluded. The full text of eligible articles was sourced and information about the intervention and population being modelled, type of modelling approach and disease monitoring strategy was extracted.

Results and discussion A total of 279 studies related to HIV simulation models were included in the review, though only $17(6 \%)$ included consideration of VL or VL testing and were evaluated in detail. Within the studies that included assessment of VL, routine monitoring was the focus, and usually in comparison to alternate monitoring strategies such as clinical or CD4 count-based monitoring. The majority of remaining models focus on the impact or delivery of antiretroviral therapy $(n=68 ; 27 \%)$, preexposure prophylaxis $(n=28 ; 11 \%$ ) and/or HIV testing $(n=24 ; 9 \%)$ on population estimates of HIV epidemiology and exclude consideration of VL. Few studies investigate or compare alternate VL monitoring frequencies, and only a small number of studies overall (3\%) include consideration of vulnerable population groups such as pregnant women or infants.

Conclusions There are very few simulations of HIV treatment or prevention that include VL measures, despite VL being recognised as the key determinant of both transmission and treatment outcomes. With growing emphasis on VL monitoring as key tool for population-level HIV control, there is a clear need for simulations of HIV epidemiology based on VL.

\section{INTRODUCTION}

Since HIV viral load (VL) was accepted as a key parameter in HIV transmission, it has become a common subject in HIV research. In addition, in 2013, the WHO recommended VL testing as the preferred antiretroviral therapy (ART) monitoring strategy. ${ }^{1}$ Mathematical models have therefore been used to determine the population-level impact

\section{Key questions}

What is already known?

- Viral load (VL) is the most important risk factor for the transmission of HIV and antiretroviral therapy (ART) is required for the suppression of HIV VL.

- VL monitoring is the preferred ART monitoring strategy and more accurately identifies poor ART adherence and ART treatment failure.

- Mathematical models are a common strategy to evaluate population impacts of interventions that have been demonstrated useful in clinical studies.

What are the new findings?

- This review of mathematical models shows that few models have been updated or created to include VL and $\mathrm{VL}$ monitoring ( $<6 \%$ include any measure of $\mathrm{VL}$ ).

- While VL monitoring is demonstrated to be an improvement over clinical or CD4 count monitoring, only monitoring strategies of biannual or annual testing were evaluated.

- Furthermore, these studies typically exclude pregnant women and other vulnerable groups from their analysis.

\section{What do the new findings imply?}

- These findings highlight the need for empirical or other studies to investigate the best timing or frequency of VL monitoring, for detection of raised VL, with interest in resource constraint settings.

- A greater variety of VL monitoring strategies should be evaluated and include key populations such as pregnant and breastfeeding women.

and feasibility of routine VL monitoring in low-resource countries. A review of all mathematical models in HIV research was therefore done, with an interest in the use of VL in these models and the findings of VL monitoring studies.

HIV-related interventions have been successful at reducing the morbidity and mortality associated with the disease, however HIV remains a public health concern. ART has played a significant role in controlling the disease, through both treatment and disease prevention. ART suppresses HIV viral 
replication, slowing disease progression, improving health and reducing HIV transmission. ${ }^{2}$ Numerous studies have identified VL as the strongest predictor of HIV transmission. ${ }^{34}$ Interim analysis of the HIV Prevention Trials Network 052 trial reported a 96\% reduction of HIV transmission risk among early ART initiators, compared with the delayed treatment patients. The preventative effect was maintained throughout the study and any HIV transmissions among the early treatment initiators were due to a viraemic episode/failure to achieve viral suppression. ${ }^{5}$ Similarly, maternal VL is directly related to the risk of vertical transmission and HIV-infected pregnant women are given lifelong antiretrovirals (ARVs) to prevent mother to child transmission (PMTCT) in subsequent and future pregnancies. ${ }^{67}$

Initially, in limited resource settings, economic constraints saw ART being scaled up in the absence of treatment monitoring. ${ }^{8-10}$ As access to treatment improved over the years, clinical and immunological monitoring of ART became important in the care of individuals living with HIV. Together, the WHO clinical criteria and CD4 measurements were used as a criterion for ART initiation and to assess treatment efficacy. ${ }^{10}$ Studies ${ }^{10-13}$ have since shown high misclassification $(43 \%-53 \%)$ and low sensitivity $(20 \%-23 \%)$ rates associated these monitoring strategies. Some patients were incorrectly identified as failing treatment and regime unnecessarily switched, while others experiencing treatment failure were switched to second-line treatment late or not at all. Remaining on failing ART has negative effects on the HIV-infected individual's health but could also increase the spread of HIV drug resistance. ${ }^{14}$ The WHO therefore recommended that VL monitoring replace the other forms of ART monitoring and that VL testing occur 6 and 12 months after ART initiation and routinely every year thereafter. ${ }^{1}$ More recently, six monthly routine VL testing has been proposed. ${ }^{15}$ The need for CD4 monitoring was further diminished as more individuals were placed on ART, regardless of any eligibility criteria and in a better health state. ${ }^{16}$ The scale-up of VL testing in low and middleincome countries (LMIC) has been slow due to the price of tests and other cost, transport and technical constraints associated with laboratory testing. ${ }^{89}$

In many LMIC settings, HIV programmes are dependent on donor funding and consequently the timing and frequency of routine VL testing can vary between developed countries and LMICs. For instance, the Department of Health and Human Services 2017 adult guidelines ${ }^{17}$ recommend VL testing 2-8 weeks immediately after ART initiation to assess viral suppression and routinely 3-4 months thereafter or 6 months for those individuals well established on ART for a duration of 2 years. In South Africa adult 2015 guidelines ${ }^{18}$ call for VL measurements at 3 and 6 months after ART initiation and if VL levels are undetectable $(<50 \mathrm{cps} / \mathrm{mL})$ then routine annual monitoring is recommended. Detectable VL results trigger a repeated test in 6 months and either adherence counselling or switching of treatment regime occurs. In severely resource-constrained settings, such as Malawi, VL measurements are recommended every 2 years for adults on stable ART (2016). ${ }^{19}$

Mathematical simulation models have long been used in infectious disease research, in instances where epidemiological studies are not feasible or ethical. ${ }^{20} 21$ They are useful for identifying potential disease control measures/interventions and to predict the future size of an epidemic or endemic. Different types of mathematical models exist, each with their individual advantages and limitations. They range from simple to complex models and are suited to investigate different research questions. Koopman ${ }^{22}$ describes the following broad set of models: compartmental models, individual/agent-based models and dynamic network models. Compartmental models, either deterministic or stochastic, are the simplest models to create and analyse. In these models, individuals behave in the same manner and the models are therefore best suited for more general estimation or prediction research questions. Individual/agent-based and dynamic network models are more realistic and complex. The populations modelled are followed individually, rather than the sum of individuals in a compartment. Dynamic network models allow for connections and links to be formed between individuals and are favoured in sexual transmission research. ${ }^{22}$ In this review, we summarise the applications of VL in the HIV mathematical models published from 2000, focusing on compartmental and individualbased models (IBM) to understand the type, features and populations that models have been applied to. We interpret and discuss the findings of VL monitoring studies and identify gaps in the literature.

\section{METHODOLOGY \\ Search strategy}

A search for relevant literature on mathematical models related to HIV/AIDS was conducted in PubMed (October 2018) using syntax constructed around the following search terms: 'HIV' and 'mathematical models'. Publications before the year 2000 were excluded, modelled chronic disease similar to HIV or did not simulate HIV data but rather analysed the data. Models on all populations, for instance, men who have sex with men (MSM) or female sex workers, were included in the review. In addition, previous reviews of HIV simulation models were included in the relevant literature and studies that involved a pharmacokinetic approach to modelling HIV or modelled the viral dynamics.

\section{Model grouping}

The full text of the relevant literature was sourced, and the characteristics of each model extracted. The following information was extracted from the literature: the intervention or the purpose of the model and contextual information including the country and population being modelled. In addition, information on the modelling approach used (compartmental vs individual, stochastic 


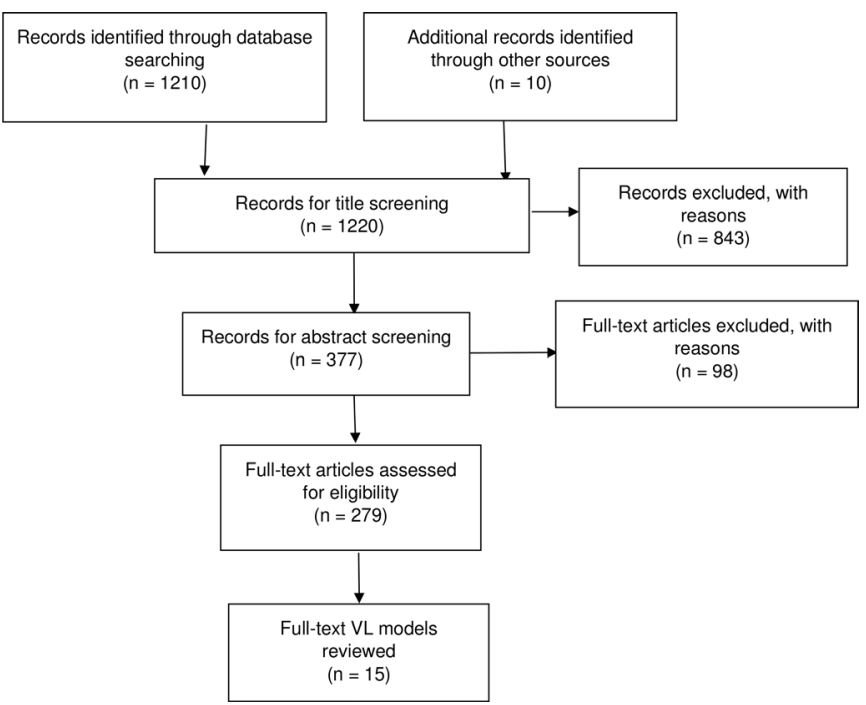

Figure 1 Preferred Reporting Items for Systematic Reviews and Meta-Analyses (PRISMA) flow diagram of studies. VL, viral load.

vs deterministic) and the inclusion of any parameters/ outcomes relating to VL were extracted.

\section{Patient and public involvement}

This review of mathematical models did not involve patients and participants.

\section{RESULTS \\ Search results}

The search yielded a total of 1136 studies and an additional 10 studies were identified through the references of the relevant articles. After title and abstract screening, 279 articles were assessed for eligibility. After applying the above listed exclusion criteria, $259 \mathrm{HIV}$ simulation/mathematical models were further assessed and the 17 studies modelling VL were included in the review (figure 1) and their results summarised in detail. The full listing of the 279 articles assessed is available as online supplementary material. A brief summary of the 259 articles evaluated is provided for some context in terms of focus and population.

A majority of simulation models focused on the delivery or impact of ART $(n=75 ; 26 \%)$, pre-exposure prophylaxis $(n=33 ; 11 \%)$ and HIV testing $(n=30 ; 10 \%)$ on population HIV burden. Most of the models were based on a heterosexual population $(n=127 ; 48 \%)$, followed by MSM ( $\mathrm{n}=40 ; 15 \%)$ and few studies included pregnant women and children $<5$ years $(n=12 ; 5 \%$ and $n=2 ;<1 \%$, respectively). Two-thirds of the global HIV burden occurs in sub-Saharan Africa (SSA) and a large portion of the simulation studies were based on data from these regions $(\mathrm{n}=123 ;<40 \%)$. This was followed by Americas and Asia ( $\mathrm{n}=32 ; 10 \%$ and $\mathrm{n}=25 ; 8 \%$, respectively).

The modelling strategy most commonly used was deterministic $(n=181 ; 65 \%)$ and population $(n=183 ; 65 \%)$ based, followed by stochastic and IBMs ( $n=54 ; 21 \%)$. Few simulation models incorporate VL in the modelling process $(n=17,6 \%)$. There were generally two types of research questions investigated in the models that included VL: (1) estimating the association between VL and HIV disease progression or ART and (2) evaluating the effectiveness and feasibility of VL monitoring strategies. The VL and HIV progression studies were mostly compartmental and estimated cohort or population VL, while the VL monitoring studies were generally stochastic IBMs that simulate individual VL trajectories.

\section{Summary of literature \\ Mathematical models with VL}

Among the 17 studies that included HIV VL, the early studies emphasised the importance of universal access to ART for successful control of the disease. These mathematical models were based on data from SSA and included VL as a measure of HIV infectivity. Using an epidemic and a patient-level model and different ART eligibility criteria, HIV incidence and prevalence were always reduced over time. In 2 years, HIV incidence was predicted to decline from $7 \%$ to $2 \%$, mortality due to HIV-related causes by $50 \%$ in 5 years and a decline in HIV prevalence $(12 \%-6 \%)$ in 11 years. ${ }^{2324}$

A more focused simulation looking at the evolution of population-level VL in SSA estimated a strong association between ART coverage and VL levels emphasising the importance of VL monitoring as the access to ART is increased. ${ }^{25}$ The cohort/population VL (on the $\log 10$ scale), simulated using a deterministic model, was unaffected by the changes in HIV prevalence and incidence over time but significantly affected by ART coverage, where even modest changes in ART coverage rate were associated with large changes in community VL. Furthermore, Estill et al showed that loss to follow-up reduces the preventative effects of ART and that tracing and follow-up of patients can be beneficial in enhancing its effects, although this decrease in the number of secondary infections was found to be modest. ${ }^{26}$

\section{VL monitoring studies}

Only $5 \%(n=13)$ of all the HIV simulation models investigated the impact of VL monitoring on the burden of HIV. All these models were based on data from SSA, $92 \%(n=12)$ were stochastic individual-type models and $77 \%(\mathrm{n}=10)$ compared VL monitoring, typically 6 and 12 months' fixed VL testing, to fixed immunological and clinical ART monitoring. There were four studies that compared routine fixed VL monitoring to other forms of ART monitoring; VL point-of-care (POC) testing, differential VL monitoring and additional drug resistance testing (online supplementary table 1 ).

All but two of the studies focused on the general adult population, ${ }^{27-35}$ there was one study investigating the optimal timing of monitoring to predict VL at delivery ${ }^{36}$ and one on the mortality among children under the age of 5 years. ${ }^{37}$ The VL monitoring studies can be summarised according to the following themes: (1) HIV incidence, drug resistance and mortality of infected individuals, (2) the vulnerable populations modelled, and (3) the future 
VL monitoring alternatives, including POC and differential VL testing (table 1).

The simulation studies showed that VL monitoring had a beneficial effect on HIV incidence, spread of drug resistance and HIV-related mortality. There were two studies that investigated the impact of routine VL monitoring on HIV incidence and both studies showed that it had a significant reduction in new HIV cases. Both simulation models were individual stochastic models that evaluated six monthly VL testing. Estill $e t a l^{28}$ compared the VL testing to biannual immunological monitoring and found an overall reduction in cohort $\mathrm{VL}$ and a $30 \%$ reduction in HIV incidence VL monitoring. Phillips $e t a l^{88}$ compared biannual immunological and three monthly clinical ART monitoring, with and without targeted/confirmatory VL testing to the VL testing. Furthermore, this strategy was also found to be cost-effective as individuals who are virally suppressed are monitored less frequently and priority given to monitoring those who fail to achieve viral suppression, therefore highlighting its potential in resource-limited settings (table 1 ).

One study showed that drug resistance was reduced in the presence of VL monitoring, thus prolonging the future use of current ARVs. Biannual and annual routine VL monitoring lowered the acquired and transmitted HIV drug resistance infections. Using a population-based compartmental model and data from Vietnam, where the prevalence of transmitted drug resistance is $5 \%-15 \%$, it was shown that even low coverage of routine VL testing can substantially reduce the prevalence of HIV drug resistance. An achievable coverage of $30 \%$ VL monitoring could potentially prevent $12 \%-31 \%$ of transmitted drug resistance (2260-5860 cases) and an estimated $25 \%-59 \%$ of acquired drug resistance (9620-22650 cases) in Vietnam ${ }^{33}$ (table 1).

The evidence supporting VL monitoring influencing mortality is less substantial. Three simulation studies have investigated the effect VL monitoring has on mortality related to HIV and found a reduction, however less pronounced and significant compared with that of HIV incidence and burden of drug resistance. All three simulations were individual stochastic models that spanned over 5 years. The first two models were based on adult populations, where Estill et al reported a modest $6 \%$ reduction in mortality with VL compared with immunological monitoring ${ }^{32}$ and Phillips et al reported similar results where $83 \%$ of potential life-years survived were predicted using VL monitoring (switch when VL $>500$ copies $/ \mathrm{mL}$ ) and $82 \%$ with immunology and clinical monitoring. ${ }^{38}$ This simulation study was however based on a population where infected individuals initiated ART at the WHO clinical stage $4 .^{38}$ Similar results were shown in a paediatric study, where the mortality over 5 years was not significantly reduced by VL monitoring when compared with CD4 monitoring. There was however an improvement in their overall CD4 measurements, more switching to second-line ART when necessary and reduced time spent on failing ART, when VL monitoring was used. ${ }^{37}$

All but one VL monitoring simulation studies excluded pregnant women from the study population and only one study focused solely on VL monitoring for this group. This model evaluated the timing of a single (assumed to be POC) VL during pregnancy to predict VL at delivery. Two timings were considered, strategies based on gestation age and strategies based on duration on ART, aiming to evaluate strategies that might be practically implemented in LMICs. The results demonstrated that taking VL measurements with respect to gestational age performed better than VL measurements based on the duration on ART. ${ }^{36}$ None of the studies included breastfeeding women.

Three mathematical models provided evidence of viable and valuable VL monitoring features for the future, POC VL testing and differential VL monitoring. A study by Estill et $a t^{28}$ aimed to estimate the cost-effectiveness of POC VL testing in resource-limited settings using a stochastic mathematical model adapted for costs and timelier returning of VL measurements. In addition, VL monitoring of 6 months was evaluated and it was found that POC VL tests were cost-effective under certain scenarios, a higher limit of detection, adherence counselling could reduce the rate of ART failure leading to a reduction in the number of new infections and/or a reduced transmission rate due to test knowledge (table 1).

Differential ART monitoring, where infected individuals with either good ART adherence or established viral suppression are monitored differently from those initiating ART or with poor adherence, may be a valuable strategy in limited resource settings. Phillips $e t a l^{29}$ used an IBM model to investigate whether there exists an opportunity for differential ART adherence monitoring through a cost-effectiveness analysis. In this model, ART adherence is modelled as the average adherence over 3 months, parameterised between $0 \%$ and $100 \%$ for each individual. Each patient's average adherence was modelled to vary over time due to factors, including: adherence interventions, age and presence of symptoms related to the toxicity of ARVs. The cost threshold for adherence monitoring was \$23-\$32 per person-year and $\$ 50$ per person-year with and without VL monitoring, respectively, and therefore differential adherence monitoring was shown to be feasible.

A second study compared fixed (1, 3, 6, 12 or 24 months) to adaptive VL monitoring strategies, to provide insight into how VL monitoring strategies should be tailored for individuals in resource-limited settings. Differentiating VL testing based on individual characteristics (reported ART adherence, age, sex and education level) resulted in less time spent on failing ART and freeing up resources. ${ }^{30}$

\section{DISCUSSION}

These mathematical models highlight the benefit of routine VL monitoring to reduce HIV incidence and the spread of HIV drug resistance. However, the results 


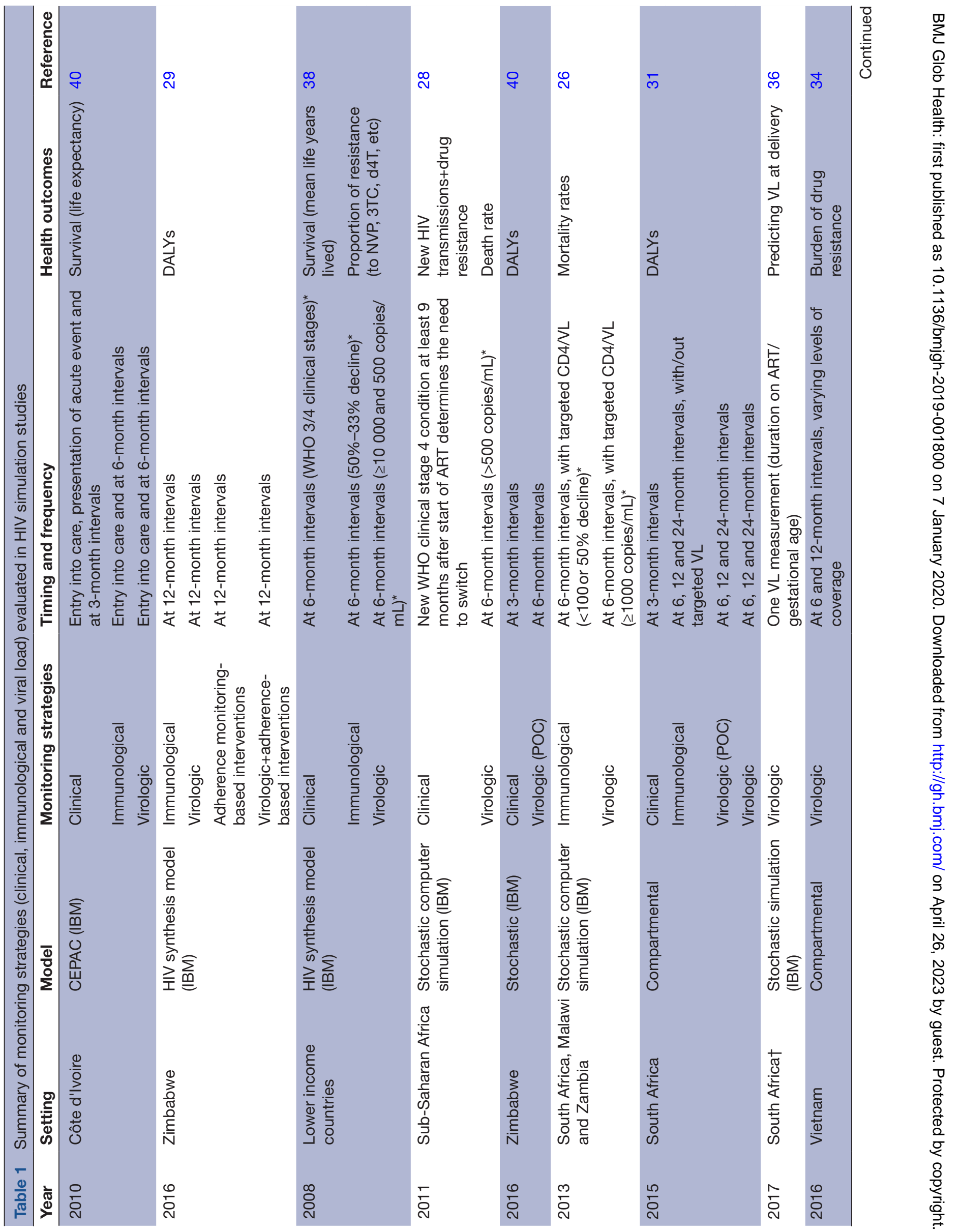




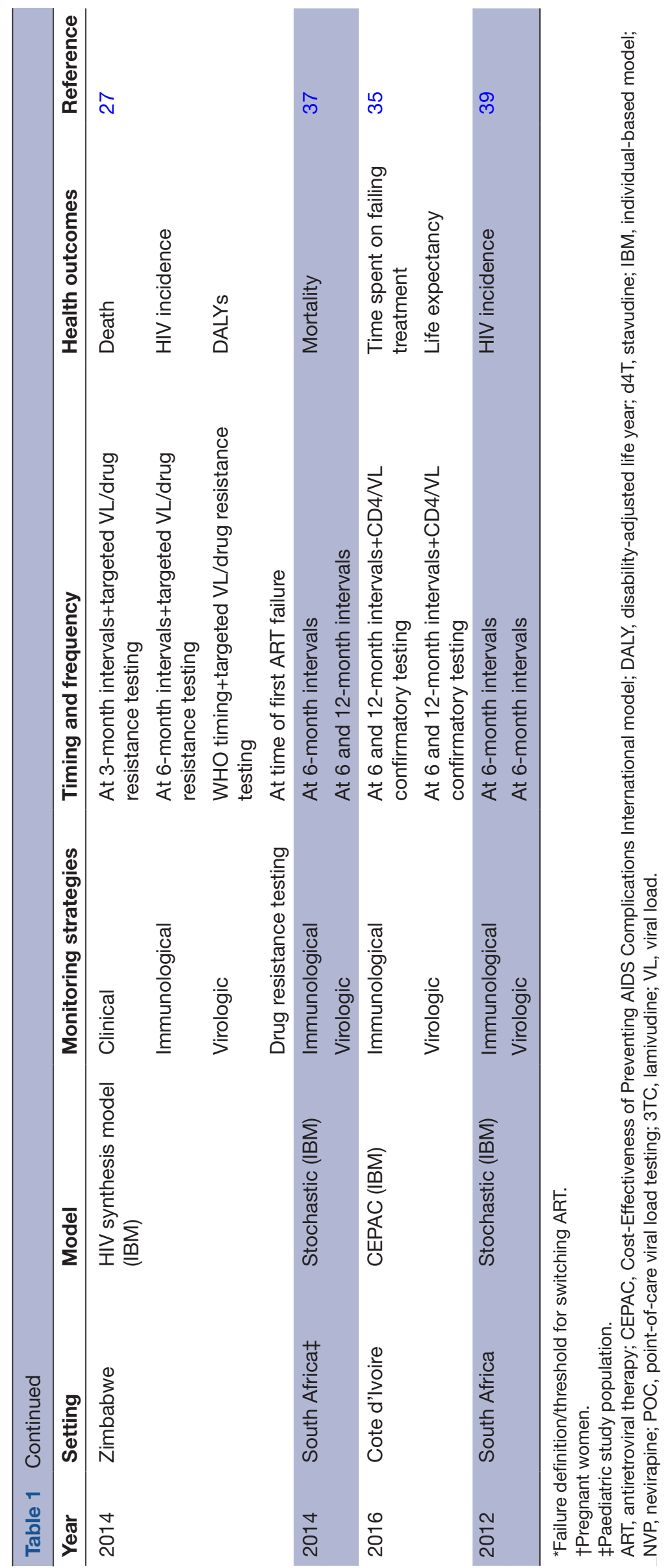

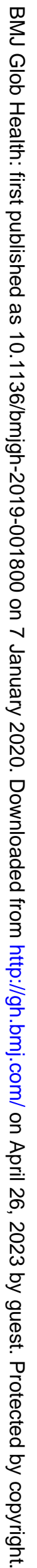


on mortality due to HIV-related causes were less robust. Despite the variety of VL testing recommendations across developing and developed settings and major policy changes over time (eg, moving to 'test and treat'), the majority of the models compared and evaluated a set strategy of either biannual or annual routine VL monitoring and in Estill $e$ t $a l \mathrm{~s}^{32}$ VL monitoring every 2 years, to one another, failing to consider novel strategies such as differentiated care. They also compare the benefits to immunological and/clinical monitoring which is rapidly being phased out. These models do not seek to determine the best timing and frequency to take VL measurements among people living with HIV, which is becoming more important as access to VL is increased in LMICs. Furthermore, as more mathematical models are developed for this purpose, it will be important to consider the features of the VL cascade, such as VL turnaround time into the modelling process. ${ }^{27-40}$

Two-thirds of the HIV burden occurs in SSA, where majority of the infection occurs in women of childbearing age. As a result, more newly pregnant women are being tested and diagnosed with HIV at antenatal clinics, where they initiate lifelong ART to PMTCT. Pregnant and breastfeeding women are therefore an important population group for HIV intervention and routine VL monitoring. ${ }^{41}$ However, the results of this review showed very few studies focus on pregnant women $(n=11,4 \%)$ and only two VL monitoring studies did not explicitly exclude pregnant women from the analysis $(<1 \%) \cdot{ }^{33}{ }^{36}$ Lesosky $e t$ $a l^{36}$ showed that if the WHO VL monitoring guidelines for adults were used, $69 \%$ of the pregnant women would not receive a VL test during pregnancy. Moreover, neither of these studies included breastfeeding women, despite the possibility of HIV transmission during this period and the recommendation by the WHO that breast feeding should be practiced, even for HIV-positive mothers in low-resource settings. In fact, studies have shown ART adherence to be problematic during the postpartum period therefore leading to increased risk of vertical transmission. $^{37}$

Furthermore, less than $1 \%$ of the mathematical models involved infants/children and only one VL monitoring study was conducted on children below the age of 5 years. ${ }^{37}$ The VL timing and frequency evaluated in this study was the same strategy used for adults, biannual and annual VL measurements which may not be appropriate for this population and different strategies should be examined. National and international policies regarding VL monitoring have been changing rapidly since the introduction of the testing and there are ongoing discussions regarding the utility, practice and scale-up of VL monitoring, as well as new technology, such as POC testing, that may change policy yet again. Mathematical modelling may be a relevant approach to evaluation of potential approaches in this context.

These mathematical models show that there is potential and benefits for the use of POC VL testing and differential ART monitoring approaches, particularly in limited resource settings. In most LMIC settings HIV care remains a 'vertical' care platform, with a single strategy for all. There are exceptions, for example, both South Africa and Kenya have differentiated care programmes for children and pregnant women. ${ }^{18}{ }^{42}$ However, there is no added benefit in additional confirmatory resistance testing when a high VL is detected using VL monitoring. ${ }^{27} 2840$

\section{CONCLUSION}

This review highlights how useful mathematical modelling has been in understanding the large-scale or longterm impact of HIV interventions. Despite this, few models have been updated or newly created to incorporate the new era of highly effective ART and routine VL monitoring. Similarly, as more empirical studies consider and call for models of differentiated care-for example-for vulnerable or special populations, the simulation models require updating. Additional work on special populations and updating or creating new models to better understand the likely impact and consequences of a rapidly changing clinical practice and policy that may be driven by health system evaluation or by donor funding are urgently needed.

Correction notice This article has been corrected since it was published. The article type has been updated.

\section{Contributors All authors contributed to the article.}

Funding TG supported by the South Africa Medical Research Council (SAMRC) National Health Scholarship Program (NHSP), South African Centre for Epidemiological Modelling and Analysis (SACEMA) Emerging Researchers Fund (ERF). The project and ML supported by Eunice Kennedy Shriver National Institute of Child Health and Human Development of the National Institutes of Health under award number R21HD093463.

Competing interests None declared.

Patient consent for publication Not required.

Provenance and peer review Not commissioned; externally peer reviewed.

Data availability statement All data relevant to the study are included in the article or uploaded as supplementary information.

Open access This is an open access article distributed in accordance with the Creative Commons Attribution Non Commercial (CC BY-NC 4.0) license, which permits others to distribute, remix, adapt, build upon this work non-commercially, and license their derivative works on different terms, provided the original work is properly cited, appropriate credit is given, any changes made indicated, and the use is non-commercial. See: http://creativecommons.org/licenses/by-nc/4.0/.

\section{REFERENCES}

1 World Health Organisation. Who consolidated guidelines on the use of antiretrovirals for treating and preventing HIV infection. Second Edition. Geneva: World Health Organisation, 2016.

2 Hull MW, Montaner JSG. Hiv treatment as prevention: the key to an AIDS-free generation. J Food Drug Anal 2013;21:S95-101.

3 Quinn TC, Wawer MJ, Sewankambo N, et al. Viral load and heterosexual transmission of human immunodeficiency virus type 1. Rakai Project Study Group. N Engl J Med 2000;342:921-9.

4 Loutfy MR, Wu W, Letchumanan M, et al. Systematic review of HIV transmission between heterosexual serodiscordant couples where the HIV-positive partner is fully suppressed on antiretroviral therapy. PLoS One 2013;8:e55747.

5 Eshleman SH, Hudelson SE, Redd AD, et al. Treatment as prevention: characterization of partner infections in the HIV prevention trials network 052 trial. J Acquir Immune Defic Syndr 2017;74:112-6. 
6 Sturt AS, Dokubo EK, Sint TT, et al. Antiretroviral therapy (art) for treating HIV infection in ART-eligible pregnant women. Cochrane Database Syst Rev 2010;22.

7 Duri K, Gumbo FZ, Kristiansen KI, et al. Antenatal HIV-1 RNA load and timing of mother to child transmission; a nested case-control study in a resource poor setting. Virol J 2010;7:176.

8 Roberts T, Cohn J, Bonner K, et al. Scale-Up of routine viral load testing in resource-poor settings: current and future implementation challenges. Clin Infect Dis 2016;62:1043-8.

9 World Health Organization. Technical and operational considerations for implementing HIV viral load testing [Accessed 14 Dec 2017].

10 Lecher S, Ellenberger D, Kim AA, et al. Scale-Up of HIV viral load monitoring - seven sub-Saharan African countries. MMWR Morb Mortal Wkly Rep 2015:64:1287-90.

11 van Oosterhout JJG, Brown L, Weigel R, et al. Diagnosis of antiretroviral therapy failure in Malawi: poor performance of clinical and immunological who criteria. Trop Med Int Health 2009;14:856-61.

12 Reynolds SJ, Nakigozi G, Newell K, et al. Failure of immunologic criteria to appropriately identify antiretroviral treatment failure in Uganda. AIDS 2009;23:697-700.

13 Rutherford GW, Anglemyer A, Easterbrook PJ, et al. Predicting treatment failure in adults and children on antiretroviral therapy: a systematic review of the performance characteristics of the 2010 who immunologic and clinical criteria for virologic failure. AIDS 2014;28:S161-9.

14 Estill J, Egger M, Johnson LF, et al. Monitoring of antiretroviral therapy and mortality in HIV programmes in Malawi, South Africa and Zambia: mathematical modelling study. PLoS One 2013;8:e57611.

15 Cohen MS, Chen YQ, World Health Organisation. What's New in Treatment Monitoring: Viral Load and CD4 Testing. Geneva: WHO 2017.

16 Ford N, Meintjes G, Vitoria M, et al. The evolving role of CD4 cell counts in HIV care. Curr Opin HIV AIDS 2017;12:123-8.

17 Panel on antiretroviral guidelines for adults and adolescents. Guidelines for the use of antiretroviral agents in adults and adolescents with HIV. Department of health and human services. Available: http://www.aidsinfo.nih.gov/ContentFiles/ AdultandAdolescentGL.pdf [Accessed 23 Mar 2019].

18 South African national department of health. National consolidated guidelines for the prevention of mother-to-child transmission of HIV (PMTCT) and the management of HIV in children, adolescents and adults. Pretoria 2015.

19 Ministry of Health, Malawi. Malawi guidelines for clinical management of HIV in children and adults. Third Edition. Lilongwe, 2016.

20 Brauer F. Epidemic models with treatment and heterogeneous mixing. Bulletin of Mathematical Biology 2008;70:1869-85.

21 Brauer F. Mathematical epidemiology: past, present, and future. Infect Dis Model 2017;2:113-27.

22 Koopman JS. Infection transmission science and models. Jpn J Infect Dis 2005;58:S3-8.

23 Granich RM, Gilks CF, Dye C, et al. Universal voluntary HIV testing with immediate antiretroviral therapy as a strategy for elimination of HIV transmission: a mathematical model. The Lancet 2009;373:48-57.

24 Palombi L, Bernava GM, Nucita A, et al. Predicting trends in HIV-1 sexual transmission in sub-Saharan Africa through the drug resource enhancement against AIDS and malnutrition model: antiretrovirals for 5 reduction of population infectivity, incidence and prevalence at the district level. Clin Infect Dis 2012;55:268-75

25 Abu-Raddad LJ, Awad SF. How does population viral load vary with the evolution of a large HIV epidemic in sub-Saharan Africa? AIDS 2014;28:927-9.
26 Estill J, Tweya H, Egger M, et al. Tracing of patients lost to followup and HIV transmission: mathematical modeling study based on 2 large art programs in Malawi. J Acquir Immune Defic Syndr 2014;65:e179-86.

27 Phillips A, Cambiano V, Nakagawa F, et al. Cost-Effectiveness of HIV drug resistance testing to inform switching to second line antiretroviral therapy in low income settings. PLoS One 2014;9:e109148.

28 Estill J, Egger M, Blaser N, et al. Cost-Effectiveness of point-of-care viral load monitoring of antiretroviral therapy in resource-limited settings: mathematical modelling study. AIDS 2013;27:1483-92.

29 Phillips A, Shroufi A, Vojnov L, et al. Sustainable HIV treatment in Africa through viral-load-informed differentiated care. Nature 2015;528:S68-76.

30 Negoescu DM, Zhang Z, Bucher HC, et al. Differentiated human immunodeficiency virus RNA monitoring in resource-limited settings: an economic analysis. Clin Infect Dis 2017;64:1724-30.

31 Phillips AN, Cambiano V, Nakagawa F, et al. Point-Of-Care viral load testing for sub-Saharan Africa: informing a target product profile. Open Forum Infect Dis 2016;3.

32 Estill J, Salazar-Vizcaya L, Blaser N, et al. The cost-effectiveness of monitoring strategies for antiretroviral therapy of HIV infected patients in resource-limited settings: software tool. PLoS One 2015; 10:e0119299.

33 Phillips AN, Pillay D, Garnett G, et al. Effect on transmission of HIV-1 resistance of timing of implementation of viral load monitoring to determine switches from first to second-line antiretroviral regimens in resource-limited settings. AIDS 2011;25:843-50.

34 Pham QD, Wilson DP, Nguyen TV, et al. Projecting the epidemiological effect, cost-effectiveness and transmission of HIV drug resistance in Vietnam associated with viral load monitoring strategies. J Antimicrob Chemother 2016;71:1367-79.

35 Ouattara EN, Robine M, Eholié SP, et al. Laboratory monitoring of antiretroviral therapy for HIV infection: cost-effectiveness and budget impact of current and novel strategies. Clin Infect Dis 2016;62:1454-62.

36 Lesosky M, Glass T, Mukonda E, et al. Optimal timing of viral load monitoring during pregnancy to predict viraemia at delivery in HIVinfected women initiating art in South Africa: a simulation study. J Int AIDS Soc 2017;20:e25000.

37 Salazar-Vizcaya L, Keiser O, Technau K, et al. Viral load versus CD4+ monitoring and 5-year outcomes of antiretroviral therapy in HIVpositive children in southern Africa. AIDS 2014;28:2451-60.

38 Phillips AN, Pillay D, Miners AH, et al. Outcomes from monitoring of patients on antiretroviral therapy in resource-limited settings with viral load, CD4 cell count, or clinical observation alone: a computer simulation model. The Lancet 2008;371:1443-51.

39 Estill $\mathrm{J}$, Aubrière $\mathrm{C}$, Egger $\mathrm{M}$, et al. Viral load monitoring of antiretroviral therapy, cohort viral load and HIV transmission in southern Africa: a mathematical modelling analysis. AIDS 2012;26:1403-13.

40 Kimmel AD, Weinstein MC, Anglaret X, et al. Laboratory monitoring to guide switching antiretroviral therapy in resource-limited settings: clinical benefits and cost-effectiveness. J Acquir Immune Defic Syndr 2010;54:258-68.

41 Myer L, Essajee S, Broyles LN, et al. Pregnant and breastfeeding women: a priority population for HIV viral load monitoring. PLOS Med 2017:14:e1002375.

42 National AIDS and STI control program, Ministry of health. Republic of Kenya improving the quality and efficiency of health services in Kenya: a practical Handbook for HIV managers and service providers on differentiated care. Nairobi, Kenya: national AIDS and STI control program, Ministry of health, 2016. http://www.differentiatedcare.org/ Portals/0/adam/Content/6ExZQGTZikegfDfhw5FSwg/File/Kenya-APractical-Handbook-for-HIV-Managers-and-Service-Providers-onDifferentiated-Care.pdf [Google Scholar] 